\title{
Is the efflux pump inhibitor Verapamil a potential booster for isoniazid against Mycobacterium tuberculosis?
}

\author{
Renata Claro Ribeiro do Amaral ${ }^{1}$, Katiany Rizzieri Caleffi-Ferracioli ${ }^{1,3^{* *}}$, \\ Fernanda de Oliveira Demitto ${ }^{2}$, Aryadne Larissa de Almeida1, \\ Vera Lucia Dias Siqueira ${ }^{1,3}$, Regiane Bertin de Lima Scodro ${ }^{2,3}$, \\ Clarice Queico Fujimura Leite ${ }^{4}$, Fernando Rogério Pavan 4 , \\ Rosilene Fressatti Cardoso ${ }^{\oplus 1,2,3^{* * * *}}$
}

\begin{abstract}
${ }^{1}$ Postgraduation in Bioscience and Physiopathology, State University of Maringa, Parana, Brazil, ${ }^{2}$ Postgraduation in Health Sciences, State University of Maringa, Parana, Brazil, ${ }^{3}$ Laboratory of Medical Bacteriology, Department of Clinical Analysis and Biomedicine, State University of Maringa, Parana, Brazil, "Laboratory of Mycobacteriology "Prof. Dr. Hugo David”, School of Pharmaceutical Science, Paulista State University, Araraquara, Sao Paulo, Brazil.
\end{abstract}

\begin{abstract}
The membrane-based efflux pump systems are recognized to have an important role in pathogenicity and drug resistance in Mycobacterium tuberculosis by the extrusion of toxic substrates and drugs from the inner bacillus. This study aimed to investigate the in vitro interaction of Verapamil (VP), an efflux pump inhibitor, with the classical first-line anti-tuberculosis drug isoniazid (INH) in resistant and susceptible $M$. tuberculosis clinical isolates. Seven multidrug-resistant (MDR), three INH monoresistant and four susceptible $M$. tuberculosis clinical isolates were tested for the INH and VP combination by modified Resazurin Microtiter Assay Plate (REMA). Fractional Inhibitory Concentration (FIC) and Modulation Factor (MF) were determined. The INH plus VP combination showed no significant change in the Minimum inhibitory concentration (MIC) values of INH (FIC $\geq$ $0.5 ; \mathrm{MF}=1$ or 2).The use of VP in tuberculosis therapy should be managed carefully, considering the resistance caused by specific mutation in $k a t G$ and inhA genes, in which the use of these EPIs may have no success. The use of EPIs as an adjunctive drug in the anti-tuberculosis therapy should be further investigated on a larger number of $M$. tuberculosis clinical isolates with different resistant profile.
\end{abstract}

Keywords: Tuberculosis. Multidrug-resistance. Efflux pumps. Efflux pumps inhibitors. Isoniazid.

\section{INTRODUCTION}

Drug resistance is a matter of great concern for tuberculosis (TB) control programs worldwide. Patients who harbor resistant TB must have alternative treatment

Correspondence: R. F. Cardoso, Departamento de Análises Clínicas e Biomedicina, Universidade Estadual de Maringá, Avenida Colombo, 5790, 87020-900, Maringá, Paraná, Brasil. Phone: +55-44-3011-5375. Fax: $+55-$ 3011-4797. E- mail: rfressatticardoso@gmail.com (R.F. Cardoso). **Both authors contributed equally to this manuscript and, in some cases, the drugs used are more expensive and toxic, and less effective (WHO, 2018).

The multidrug-resistant (MDR) Mycobacterium tuberculosis phenotype is caused by sequential mutations in specific chromosomal genes, which are related to the mechanism of rifampicin (RIF) and isoniazid (INH) actions (Zhang, Yew, 2009). However, the genetic basis of these two and other anti-TB drugs resistance are not fully known. In some resistant bacilli, the classical mutations related to resistance to specific drugs are not present, suggesting other resistance mechanisms 
are responsible for the resistant phenotype (Ghajavand et al., 2019).

At present, the membrane-based efflux pump systems (EPs) are recognized to have an important role in the bacterial pathogenicity and antimicrobial resistance, including in M. tuberculosis, by the extrusion of toxic substrates and drugs from the inner cell. Nonetheless, not only have EPs important biological role in drug extrusion, but also in the uptake of substrates and nutrients necessary to the bacillus survival (Gupta et al., 2010a; Ghajavand et al., 2019; Rodrigues et al., 2012; Chen et al., 2018).

These transporters can be classified as primary active transporters, which use ATP direct energy to promote the transport of molecules across the bacterial membrane, against their concentration gradient. The secondary active transporters are membrane proteins that use an electrochemical gradient of cations, the proton motive force, across the membrane coupled to drug extrusion against its concentration gradient (Sharma, Gupta, Pathania, 2019).

Verapamil (VP), a calcium channel blocker, is a drug commonly used to treat hypertension and has shown to have activity as EPs inhibitors (EPI) in $M$. tuberculosis (Machado et al., 2012). More recently, the M. tuberculosis's membrane pharmacologic target mechanistic basis for the role of verapamil's potentiation of TB drugs was recognized as having the ability to directly impact on membrane energetic (Chen et al., 2018).

In the search for new treatments in cases of resistant $\mathrm{TB}$, in which classical anti-TB drugs are not effective, the combinatory use of efflux pumps inhibitors (EPI), such as VP, has become the subject of some studies (Gupta et al., 2010a; Rodrigues et al., 2012; Cloete et al., 2018; Sharma et al., 2010). However, the experimental procedures for understanding EPI activity in $M$. tuberculosis are mostly limited to laboratory strains and few have been performed with clinical isolates (Machado et al., 2012), and divergence in results have been observed. Our study aimed to know how the combination of VP with the classical first-line anti-TB drug INH has activity, in vitro, against susceptible and resistant (including MDR) $M$. tuberculosis clinical isolates.

\section{MATERIAL AND METHODS}

\section{Clinical isolates}

A total of 14 resistant and susceptible $M$. tuberculosis clinical isolates (seven MDR, three INH monoresistant and four susceptible to the anti-TB firstline drugs), previously determined by proportion method in Löwenstein-Jensen and genotyped by Mycobacterial Interspersed Repetitive Unit - Variable Number Tandem Repeat (MIRU-VNTR) (Supply et al., 2001) and Spoligotyping (Molhuizemet al.,1998), were selected for the study (Table I). M. tuberculosis wild type 37: subscribed (ATCC 27294) strain was used in all assays as control. Sequencing reactions, to determine mutation in inh $A$ regulatory and inhA and $k a t G$ structural genes, were conducted previously (Cardoso et al., 2004).

\section{Minimum inhibitory concentration (MIC)}

The MIC of INH and VP (Sigma-Aldrich, St. Louis, MO, USA) were determined, three times on different days for $M$. tuberculosis $H_{37} R v$ and all clinical isolates by REMA (Resazurin Microtiter Assay Plate), as previously described (Palomino et al., 2002). Carbonyl cyanide m-chlorophenyl-hydrazone (CCCP), a strong protonophore, which specifically dissipates the transmembrane proton gradient component of the proton motive force, was used as EPI reference drug (Chen et al., 2018). The drugs concentrations ranged from 0.0009 to $50.0 \mu \mathrm{g} / \mathrm{mL}, 3.90$ to $500.0 \mu \mathrm{g} / \mathrm{mL}$ and 0.39 to $100.0 \mu \mathrm{g} / \mathrm{mL}$ for INH, VP and CCCP, respectively. Growth and sterility controls for $M$. tuberculosis $H_{37} R v$ and all clinical isolates were carried out in all assays. MIC was defined as the lowest drug concentration that prevented a color change of resazurin from blue to pink. Isolates with $\mathrm{MIC} \geq 0.25 \mu \mathrm{g} /$ $\mathrm{mL}$ were considered INH resistant (Palomino et al., 2002).

\section{Drugs Combination assay}

The combination of VP and CCCP with INH were performed using VP and CCCP at constant concentration ( $1 / 2 \times$ MIC previously determined by REMA). For the assay, $100 \mu \mathrm{L}$ of OADC-supplemented Middlebrook 7H9 (Difco Laboratories, Detroit, MI, USA) were added to each well in the microtiter plates and two-fold serial dilutions of INH were carried out. Mycobacterial inoculums were standardized with 1 McFarland scale and diluted (1:20) in OADC-supplemented Middlebrook 
TABLE I - Mycobacterial Interspersed Repetitive Unit (MIRU) and Spoligotyping patterns, drugs susceptibility profiles, katG and inhA mutations in Mycobacterium tuberculosis $H_{37} R v$ and clinical isolates

\begin{tabular}{|c|c|c|c|c|c|}
\hline \multirow{2}{*}{ Strain/Isolates } & \multirow{2}{*}{ MIRU } & \multirow{2}{*}{ Spoligotyping } & \multirow{2}{*}{ Susceptibility pattern } & \multicolumn{2}{|c|}{ Mutation } \\
\hline & & & & $k a t G$ & $\operatorname{inh} A$ \\
\hline $\mathrm{H}_{37} \mathrm{Rv}$ & - & - & S & - & - \\
\hline $9 \mathrm{~S}$ & - & - & S & - & - \\
\hline 46 & 224326132323 & 777777774020771 & S & - & - \\
\hline 47 & 123323132321 & 777777777760771 & S & - & - \\
\hline 25252 & - & 000000007760771 & S & - & - \\
\hline $34 \mathrm{R}$ & 122222153321 & 777777777760771 & $\mathrm{INH}^{\mathrm{R}}$ & Ser 315Thr & NM \\
\hline $91 \mathrm{R}$ & - & 677737607760771 & $\mathrm{INH}^{\mathrm{R}}$ & Ser 315Thr & NM \\
\hline 4250 & - & 777777777760710 & $\mathrm{INH}^{\mathrm{R}}$ & Ser315Ile & NM \\
\hline $64 \mathrm{~A}$ & 124325163322 & 677737607760771 & $\mathrm{INH}^{\mathrm{R}} / \mathrm{RIF}^{\mathrm{R}}$ & NM & $\mathrm{c}$ to $\mathrm{t}$ at -15 ; Ile21Thr \\
\hline $73 \mathrm{~A}$ & 224225123321 & 677737607760771 & $\mathrm{INH}^{\mathrm{R}} / \mathrm{RIF}^{\mathrm{R}} / \mathrm{PZA}^{\mathrm{R}}$ & Ser315Thr & NM \\
\hline 109 & 224326153325 & 776177607760771 & $\mathrm{INH}^{\mathrm{R}} / \mathrm{RIF}^{\mathrm{R}}$ & Ala109Val & NM \\
\hline 18 & 224326153324 & 776177607760771 & $\mathrm{INH}^{\mathrm{R}} / \mathrm{RIF}^{\mathrm{R}} / \mathrm{EMB}^{\mathrm{R}}$ & Ser315Thr & NM \\
\hline 19 & 224327153324 & 776177607760771 & $\mathrm{INH}^{\mathrm{R}} / \mathrm{RIF}^{\mathrm{R}} / \mathrm{EMB}^{\mathrm{R}}$ & Ser315Thr & NM \\
\hline $71 \mathrm{~A}$ & 225313153323 & 777777777720771 & $\mathrm{INH}^{\mathrm{R}} / \mathrm{RIF}^{\mathrm{R}} / \mathrm{EMB}^{\mathrm{R}}$ & Ser315Thr & NM \\
\hline 3614 & 224225163321 & 677737607760771 & $\begin{array}{c}\text { INHR/RIFR/EMBR } \\
\text { ETHR }^{\mathrm{R}}, \mathrm{STR}^{\mathrm{R}}\end{array}$ & NM & $\mathrm{c}$ to $\mathrm{t}$ at -15 ; Ile21Thr \\
\hline
\end{tabular}

S, susceptible; R, resistant; INH, isoniazid; RIF, rifampicin; EMB, ethambutol; PZA, Pyrazinamide; STR: streptomycin; ETH: ethionamide $k a t G$, gene that encode Catalase peroxidase enzyme; inhA, gene that encode the enoyl ACP reductase NADH dependent enzyme; -, not performed; NM, no mutation detected. 
$7 \mathrm{H} 9$ (BBL/Becton Dickinson, Sparks, MD, USA) added of VP or CCCP to achieve $1 / 2 \times$ MIC final concentration in all microplates wells. The final concentration of INH in the microplates wells ranged from 0.0009 to $50 \mu \mathrm{g} /$ $\mathrm{mL}$. Growth and sterility controls for M. tuberculosis $H_{37} R v$ and all clinical isolates were carried out in all assays. Incubation conditions and reading were carried out according to REMA (Palomino et al., 2002).

The effect of VP and CCCP on the activity of INH were determined by the fractional inhibitory concentration (FIC) according to Pillai et al. (2005), where $\mathrm{FIC}=\mathrm{MIC}$ drugs combination/MIC INH. The results were interpreted according to Coelho et al. (2012), considering FIC $\leq 0.25$ as synergism, FIC $>0.25$ $<2$ as indifferent, and FIC $\geq 2$ as antagonism. To quantify the effect of each inhibitor (VP and CCCP) in reducing the INH MIC values, the modulation factor (MF) was applied by dividing the MIC value determined for INH by the MIC obtained with the INH plus EPI combination (MF=MIC INH/MIC INH+EPI). A four-fold reduction on the MIC value of the INH combined with VP or CCCP (MF value $\geq 4$ ) was considered significant for INH in having better action against the bacillus.

\section{RESULTS AND DISCUSSION}

Table II shows MIC values of INH, VP, CCCP and INH combined with the two EPIs at constant concentration for M. tuberculosis $H_{37} R v$ and all studied isolates. All isolates were genetically differentiated and the presence of mutation in specific genes related with resistance to INH was known. The resistant isolates that had Ser315Thr or Ser315Ile mutations in the Catalase enzyme (kat $G$ ) showed MIC range from 3.125 to 12.5 $\mu \mathrm{g} / \mathrm{mL}$. The isolate with Ser 109 Val mutation in $\mathrm{katG}$ had lower INH MIC, $1.56 \mu \mathrm{g} / \mathrm{mL}$. These MIC values, obtained in our study, corroborate with the literature inferring resistance to INH in $M$. tuberculosis. The cumulative mutations in the regulatory region and in the open reading frame of inhA certainly could induce the MIC value observed for the two INH resistant isolates 64A and 3614 (Walker et al., 2015).

Analyzing the MIC of VP and CCCP, in susceptible and in resistant isolates, lower values were detected for CCCP than VP. This assay was previously carried out for all isolates to determine the $1 / 2 \times$ MIC concentration to perform the drugs combination assays.

As far as we have knowledge, both VP and CCCP are inhibitors of secondary active transporters. This kind of transporters act extruding different compounds by using an electrochemical gradient of cations, the proton motive force, across the cell membrane inducing extrusion against its concentration gradient. VP can modify the proton-motive force of the cell membrane, which is an essential requirement for the function of specifics EPs. Then, VP blocks the activity of EPs and the concentration of antimicrobial agents inside the cells will be higher and may cause bacterial death (van Bambeke, Balzi, Tulkens, 2000).

The effects of VP and other EPIs on reducing the drug resistance in $M$. tuberculosis, have been previously reported (Gupta et al., 2010b; Rodrigues et al., 2012; Cloete et al., 2018; Machado et al., 2012; Sharma et al., 2010). However, we must emphasize that the M. tuberculosis isolates in the above studies were laboratory-induced resistant mutants, which showed EPs (over)expression under drug pressure status, such as prior bacillus exposure to sub-inhibitory concentrations of drugs (Rodrigues et al., 2012; Sharma et al., 2010).

Like other studies (Coelho et al., 2015; Machado et al., 2016), the combination of VP with INH in our work showed no significant change in the MIC values of INH (FIC $\geq 0.5 ; \mathrm{MF}=1$ or 2 ) in the studied clinical isolates (Table II). Coelho et al. (2015), working with clinical isolates, observed a $\mathrm{MF}=4$ only for the pan susceptible reference strain $\mathrm{H}_{37} \mathrm{Rv}$, which had an initial MIC $0.1 \mu \mathrm{g} / \mathrm{mL}$ for INH and, after the addition of VP at $1 / 4 \mathrm{MIC}$ concentration, the MIC value for INH decreased to $0.025 \mu \mathrm{g} / \mathrm{mL}$. In our study, the used $\mathrm{H}_{37} \mathrm{Rv}$ reference strain showed a lower initial MIC $0.06 \mu \mathrm{g} / \mathrm{mL}$, and no decrease in this value after the addition of VP at $1 / 2$ MIC concentration was observed. Machado et al. (2016) in the same field of study, observed excellent action of $\mathrm{VP}$ in decreasing the MIC of INH in only one resistant isolate, which had a MF $=20$. No interference of VP, in this sense, was observed in the susceptible $\mathrm{H}_{37} \mathrm{Rv}$ and in the INH resistant $\mathrm{H}_{37} \mathrm{Rv}$ mutant. Of course, we could not fail to see, in the above study, a little decrease in the INH MICs values in the other isolates, which account for a $\mathrm{MF}=3$ in three resistant isolates.

Despite the limitation of using a low number of clinical isolates in our study, the addition of VP at $1 / 2 \times$ MIC concentration did not enhance the INH activity in all $M$. tuberculosis (FIC $\geq 0.5 ; \mathrm{MF}=1$ and 2) studied. The result of no significant reduction of the INH MIC by the combination with VP might be due to the studied isolates, which had specific mutation in the $\operatorname{kat} G$ or inhA genes. Our data corroborates with 
TABLE II - Minimum inhibitory concentration (MIC) of Isoniazid and efflux pump inhibitors alone and in combination with $1 / 2$ x MIC of efflux pump inhibitors, Fractional Inhibitory Concentration (FIC) and Modulation Factor (MF) for Mycobacterium tuberculosis $\mathrm{H}_{37} \mathrm{Rv}$ and clinical isolates

\begin{tabular}{|c|c|c|c|c|c|c|c|c|c|}
\hline \multirow{2}{*}{$\begin{array}{l}\text { Strain/ } \\
\text { Isolates }\end{array}$} & \multicolumn{3}{|c|}{$\begin{array}{c}\text { REMA } \\
\text { MIC }(\mu \mathrm{g} / \mathrm{mL})\end{array}$} & \multicolumn{2}{|c|}{$\begin{array}{c}\text { REMA } \\
\text { MIC }(\mu \mathrm{g} / \mathrm{mL})\end{array}$} & \multicolumn{2}{|c|}{ FIC } & \multicolumn{2}{|c|}{ MF } \\
\hline & СССР & VP & INH & INH+CCCP & INH+VP & $\mathrm{INH}+\mathrm{CCCP}$ & INH+VP & INH+CCCP & INH+VP \\
\hline $\mathrm{H}_{37} \mathrm{Rv}$ & 3.125 & 125 & 0.06 & 0.03 & 0.03 & 0.5 & 0.5 & 2 & 2 \\
\hline 9S & 1.56 & 62.5 & 0.06 & 0.03 & 0.03 & 0.5 & 0.5 & 2 & 2 \\
\hline 46 & 1.56 & 125 & 0.03 & 0.03 & 0.03 & 1 & 1 & 1 & 1 \\
\hline 47 & 3.125 & 250 & 0.06 & 0.06 & 0.03 & 1 & 0.5 & 1 & 2 \\
\hline 25252 & 1.56 & 62.5 & 0.125 & 0.06 & 0.125 & 0.48 & 1 & 2 & 1 \\
\hline $34 \mathrm{R}$ & 3.125 & 125 & 6.25 & 3.125 & 6.125 & 0.5 & 1 & 2 & 1 \\
\hline $91 \mathrm{R}$ & 1.56 & 62.5 & 12.5 & 12.5 & 12.5 & 1 & 1 & 1 & 1 \\
\hline 4250 & 1.56 & 125 & 6.25 & 6.25 & 3.125 & 1 & 0.5 & 1 & 2 \\
\hline $64 \mathrm{~A}$ & 1.56 & 125 & 12.5 & 6.25 & 6.25 & 0.5 & 0.5 & 2 & 2 \\
\hline $73 \mathrm{~A}$ & 3.125 & 125 & 6.25 & 3.125 & 3.125 & 0.5 & 0.5 & 2 & 2 \\
\hline 109 & 1.56 & 125 & 1.56 & 1.56 & 1.56 & 1 & 1 & 1 & 1 \\
\hline 18 & 0.78 & 125 & 3.125 & 3.125 & 3.125 & 1 & 1 & 1 & 1 \\
\hline 19 & 1.56 & 62.5 & 3.125 & 1.56 & 3.125 & 0.5 & 1 & 2 & 1 \\
\hline $71 \mathrm{~A}$ & 3.125 & 125 & 12.5 & 6.25 & 6.25 & 0.5 & 0.5 & 2 & 2 \\
\hline 3614 & 3.125 & 62.5 & 6.25 & 6.25 & 3.125 & 1 & 0.5 & 1 & 2 \\
\hline
\end{tabular}

INH, isoniazid; VP, verapamil; CCCP, Carbonyl cyanide $m$-chlorophenyl-hydrazone. REMA: Resazurin Microtiter Assay Plate.

Machado et al. (2016), who found a limited effect of VP and other EPIs in isolates harboring mutation in inh $A$ and little or no effect in isolates with mutation in $k a t G$, in which those mutations can induce low and high level resistance, respectively.

Although our and other in vitro studies have shown low effectiveness of the INH and VP combination in some $M$. tuberculosis isolates, studies in animal model, combining VP at a dosage of $6.25 \mathrm{mg} / \mathrm{kg}$ restored the susceptibility to this classical first-line anti-TB drug in mice infected with MDR M. tuberculosis (Louw et al., 2011). Additionally, an in vitro study with $M$. tuberculosis infected macrophages (Machado et al., 2016) showed EPIs cause alterations in the transport of cations in eukaryotic vacuolar efflux pumps and enhancement of the macrophages mediated the killing of the bacillus. This effect in the macrophage associated with the proposed direct interference of the EPIs, as VP, 
on the bacterial cell energy (Black et al., 2014, Chen et al., 2018) could improve the TB therapy.

In the opinion of these authors, there is no doubt on EPs role as an alternative mechanism for anti-TB drug resistance in some $M$. tuberculosis clinical isolates, or even influence to acquire other resistance mechanisms. However, a consideration on the lack of influence of VP on restoring INH susceptibility in some MDR $M$. tuberculosis isolates is clear in our study. The resistance in these isolates is consequence of mutations in the kat $G$ and inhA genes and, even VP having the ability to directly impact bacillus membrane energetics, no synergism with INH plus VP could be observed.

As no effect in decreasing the MIC of INH by the addition of VP, including with our EPI control, CCCP, was observed in our study with susceptible and resistant clinical isolates, it is clear that the activity of VP in the acquisition, as well as in the maintenance of INH resistance in the $M$. tuberculosis clinical isolates studied is only one of the involved mechanisms, as reported by Song and $\mathrm{Wu}$ (2016). Then, the consideration of efflux activity and the use of EPI in therapy of TB still need further studies on a larger number of MDR M. tuberculosis isolates with different resistant genetic profiles. The use of VP in the therapy of TB should be managed carefully, considering the resistance caused by specific mutations in kat $G$ and inh $A$ genes or other genes, which may lead to the failure of the use of these EPIs. The use of EPIs as adjunctive anti-TB therapy should be more investigated and the issue of their activity in infected macrophage and in reducing anti-TB drugs side effects considered.

\section{ACKNOWLEDGEMENTS}

We are grateful to Laboratório de Bacteriologia Médica, belonging to Laboratório de Ensino e Pesquisa em Análises Clínicas (LEPAC), Coordenação de Aperfeiçoamento de Pessoal de Nível Superior (CAPES) and Conselho Nacional de Desenvolvimento Científico e Tecnológico (CNPq), Brazil.

\section{Funding}

No funding.

\section{Ethical approval}

Not required.

\section{Competing interest}

The authors declare that they have no competing interest.

\section{REFERENCES}

Black PA, Warren R, Louw GE, Van Helden PD, Victor TC, Kana BD. Energy metabolism and drug efflux in Mycobacterium tuberculosis. Antimicrob Agents Chemother. 2014;58(5):2491-503.

Cardoso RF, Cooksey RC, Morlock GP, Barco P, Cecon $\mathrm{L}$, Forestiero $\mathrm{F}$ et al. Screening and characterization of mutations in isoniazid-resistant Mycobacterium tuberculosis isolates obtained in Brazil. Antimicrob Agents Chemother. 2004;48(9):3373-81.

Chen C, Gardete S, Jansen RS, Shetty A, Dick T, Rhee KY et al.Verapamil targets membrane energetics in Mycobacterium tuberculosis.Antimicrob. Agents Chemother. 2018; doi:10.1128/ AAC.02107-17.

Cloete R, Kapp E, Joubert J, Christoffels A, Malan SF. Molecular modelling and simulation studies of the Mycobacterium tuberculosis multidrug efflux pump protein Rv1258c. PLoS One. 2018;13(11):e0207605; doi:10.1371/ journal.pone.0207605

Coelho T, Machado D, Couto I, Maschmann R, Ramos D, Von Groll A et al. Enhancement of antibiotic activity by efflux inhibitors against multidrug resistant Mycobacterium tuberculosis clinical isolates from Brazil. Frontiers in Microbiology. 2015; 6:330.

Ghajavand H, Kargarpour Kamakoli M, Khanipour S, et al. Scrutinizing the drug resistance mechanism of multi- and extensively-drug resistant Mycobacterium tuberculosis: mutations versus efflux pumps. Antimicrob Resist Infect Control. 2019;8:70.

Gupta AK, Reddy VP, Lavania M, Chuahan DS, Venkatesan $\mathrm{K}$, Sharma VD et al. jefA (Rv2459), a drug efflux gene in Mycobacterium tuberculosis confers resistance to isoniazid \& ethambutol. Indian J Med Res. 2010a;132:176-188.

Gupta AK, Katoch VM, Chauhan DS, Sharma R, Singh M, Venkatesan K et al. Microarray analysis of efflux pump genes in multidrug-resistant Mycobacterium tuberculosis during stress induced by common anti-tuberculous drugs. Microb Drug Resist. 2010b;16(1):21-28.

Louw GE, Warren RM, Gey Van Pittius NC, Leon R, Jimenez A, Hernandez-Pando R et al. Rifampicin Reduces 
Is the efflux pump inhibitor Verapamil a potential booster for isoniazid against Mycobacterium tuberculosis?

Susceptibility to Ofloxacin in Rifampicin resistant Mycobacterium tuberculosis through Efflux. Am J Respir Crit Care Med. 2011;184(2):269-76.

Machado D, Couto I, Perdigão J, Rodrigues L, Portugal I, Baptista $\mathrm{P}$ et al. Contribution of efflux to the emergence of isoniazid and multidrug resistance in Mycobacterium tuberculosis. PloS One. 2012;7(4):34538.

Machado D, Pires D, Perdigão J, Couto I, Potugal I, Martins $\mathrm{M}$ et al. Ion channel blockers as antimicrobial agents, efflux inhibitors, and enhancers of macrophage killing activity against drug resistant Mycobacterium tuberculosis. PloS One. 2016;11(2):e0149326.

Molhuizem HOF, Bunschoeten AE, Schouls LM, Van Embden JDA. Rapid detection and simultaneous strain differentiation of Mycobacterium tuberculosis complex bacteria by spoligotyping. Methods Mol Biol. 1998;101:381-394.

Palomino JC, Martin A, Camacho M, Guerra H, Swings J, Portaels F. Resazurin microtiter assay plate: simple and inexpensive method for detection of drug resistance in Mycobacterium tuberculosis. Antimicrob Agents Chemother. 2002;46(8):2720-2722.

Pillai S, Moellering R, Eliopoulos GM. Antimicrobial combinations. 5th ed. New York: Lippincott Williams \& Wilkins, 2005.

Rodrigues L, Machado D, Couto I, Amaral L, Viveiros M. Contribution of efflux activity to isoniazid resistance in the Mycobacterium tuberculosis complex. Infect Genet Evol. 2012;12(4):695-700.
Sharma S, Kumar M, Sharma S, Nargotra A, Koul S, Khan IA. Piperine as an inhibitor of Rv1258c, a putative multidrug efflux pumps of Mycobacterium tuberculosis. J Antimicrob Chemother. 2010;65(8):1694-701.

Sharma A, Gupta VK, Pathania R. Efflux pump inhibitors for bacterial pathogens: From bench to bedside. Indian J Med Res. 2019;149(2):129-145.

Song L, Wu X. Development of efflux pump inhibitors in antituberculosis therapy. Int $\mathrm{J}$ Antimicrob Agents. 2016;47(6):421-9.

Supply P, Lesjean S, Savine E, Kremer K, Van Soolingen D, Loucht C. Automated high-throughput genotyping for study of global epidemiology of Mycobacterium tuberculosis based on mycobacterial interspersed repetitive units. J Clin Microbiol. 2001;39(10):3563-3571.

Van Bambeke F, Balzi E, Tulkens PM. Antibiotic efflux pumps. Biochem Pharmacol. 2000;60(4):457-70.

Zhang Y, Yew WW. Mechanisms of drug resistance in Mycobacterium tuberculosis. Int $\mathrm{J}$ Tuberc Lung Dis. 2009;13(11):1320-30.

Walker TM, Kohl TA, Omar SV, Hedge J, Del Ojo Elias C, Bradley $\mathrm{P}$ et al. Whole-genome sequencing for prediction of Mycobacterium tuberculosis drug susceptibility and resistance: a retrospective cohort study. Lancet Infect Dis. 2015;15(10):1193-202.

World Health Organization. Global tuberculosis report 2018. Geneva: 2018.

Received for publication on $19^{\text {th }}$ April 2018 Accepted for publication on $05^{\text {th }}$ August 2019 